\title{
Differential Diagnosis of Vertigo in Children
}

\author{
Anita Bhandari ${ }^{1}$ Arpana Goswami ${ }^{1}$ \\ ${ }^{1}$ Department of ENT, Vertigo and Ear Clinic, Jaipur, Rajasthan, India
}

Ann Otol Neurotol ISO 2019;2:21-26

\begin{abstract}
Address for correspondence Anita Bhandari, MS (ENT), Department of ENT, Vertigo and Ear Clinic, Mahaveer Nagar, Jaipur 302018 , Rajasthan, India (e-mail: dranitabhandari@gmail.com).
\end{abstract}

\begin{abstract}
Keywords

- pediatric vertigo

- vestibular disorders

- clinical characteristics

- differential diagnosis

Introduction Vertigo and balance disorders can affect all age groups. It is common to misdiagnose the cause of dizziness in children, which eventually results in the delay of treatment. The purpose of this study was to review the clinical characteristics and neurotological evaluation of vertigo in patients younger than 16 years and assist in making a differential diagnosis for children with vertigo and balance disorders. The differential diagnosis can help determine the correct management strategy to treat vertigo and imbalance in pediatric patients.

Methods Children and adolescents presenting with a chief complaint of vertigo or unsteadiness to Vertigo and Ear Clinic, Jaipur, were selected for this study on the basis of clinical history, physical examination, and neurotological evaluation.

Results A total of 90 patients with a mean age of 10.67 years were evaluated. The most common cause associated with vertigo or dizziness was found to be vestibular migraine seen in 38 participants of this study. This was followed by unilateral peripheral vestibulopathy in 15 patients, bilateral peripheral vestibulopathy in 11 patients, and central vestibulopathy in 6 patients. Vertigo was also seen to be associated with various other disorders.

Conclusion Vertigo in children must be recognized as a definite symptom, which should be taken seriously by the clinician and family. Pediatric vertigo can be due to many etiologies. History and evaluation are the only way to diagnose the etiology and site of lesion. This will prevent misdiagnosis and delay in the treatment, which can ultimately lead to deterioration of the quality of life.
\end{abstract}

\section{Introduction}

Vertigo and dizziness are not uncommon during childhood even though it is often perceived as a disorder of older people. According to a study on Scottish school children, 15\% were seen to have at least one episode of dizziness over a period of 1 year. ${ }^{1}$ Many other studies show a high prevalence, but it is still an area that needs to be explored further. Approach to vertigo in children is not easy due to the difficulty in eliciting a clear and reliable history and parents often suspecting that the children are making excuses for not going to school, thereby ignoring the symptoms of vertigo. Pediatric vertigo was first described by Harrison in $1962 .{ }^{2}$ Our understanding of vestibular disorders, along with better tools of evaluation, has brought about a tremendous change in our approach to patients with vertigo and balance the disorder since then. After a detailed history, clinical examination and neurotological evaluation, the diagnosis and required form of management become clearly delineated. A child presenting with dizziness is usually examined by a general practitioner or a pediatrician. It is very common to misdiagnose the cause of dizziness in children, which eventually results in the delay of the treatment. ${ }^{3}$

Vertigo in children could be seen due to many reasons. These include trauma, infections, migraine, disorders of the central nervous system (usually cerebellar and brainstem), benign paroxysmal positional vertigo (BPPV), vestibular neuritis due to the viral infections, and psychosomatic disorders. ${ }^{4}$ Of these, otitis media and middle ear infusion are found to be the most common causes of vestibular disturbances in

\section{received}

April 23, 2019

accepted

May 16, 2019

published online

July 16, 2019
Copyright @2019 Indian Society of Otology
License terms

(요 (1) $\odot$ 
children. ${ }^{4,5}$ It is important to develop a differential diagnosis of vertigo in children with normal tympanic membrane. ${ }^{6}$ Vertigo in children is different from that seen in adults due to three main reasons. First reason is difficulty in eliciting the detailed history as children may be unable to describe the symptoms in detail. Pertinent clinical history and careful examination are the only ways to achieve early and accurate diagnosis. Second is the fact that parents and often clinicians are unaware of vertigo being a presenting complaint in children, which leads to erroneous impressions that the resulting symptoms could be related to behavioral problems or incoordination. Finally, although most of the diseases causing vertigo are common both in children and adults, the frequency is less in children in comparison to the adults (e.g., BPPV). ${ }^{7}$ Furthermore, pediatric patients present a big challenge to vestibular examination. Static vestibular deficits are difficult to diagnose in them. Many vestibular problems leading to disequilibrium in adults are not seen in pediatric patients. ${ }^{3}$

The purpose of this study was to review the clinical characteristics and neurotological findings in patients presenting with vertigo or dizziness younger than 16 years and assist in making a differential diagnosis.

\section{Methodology}

A total of 90 children (16 years and less) coming to Vertigo and Ear Clinic, Jaipur, with a chief complaint of vertigo or dizziness within a period of 1 year were selected for this study on the basis of clinical history, physical examination, and neurotological evaluation. Participants with abnormal tympanograms and chronic suppurative otitis media (CSOM) were excluded from this study. Also, the participants who did not perform the vestibular or audiological evaluations were not included. A detailed history of attacks of vertigo, their duration, triggers, associated symptoms, drug history, and family history were obtained. All participants were evaluated with various neurotological tests such as video nystagmography (VNG), craniocorpography (CCG), computerized dynamic visual acuity (DVA), subjective visual vertical (SVV), and audiometry. Computerized DVA was not done in children younger than 6 years.

\section{Results}

The average age of patients was 10.67 years with a standard deviation (SD) of 4.35, ranging from a minimum of 3 years to a maximum of 16 years. Fifty-six study participants were males and 34 were females. - Table 1 shows the demographic characteristics of all the study participants.

-Fig. 1 shows the distribution of the disorder in the study participants. The most common cause identified for vertigo was vestibular migraine seen in 38 (42.22\%) patients. This was followed by unilateral peripheral vestibulopathy seen in 15 (16.67\%) patients, bilateral peripheral vestibulopathy in $11(12.22 \%)$ patients, and central vestibulopathy in $6(6.67 \%)$ pateints. The other disorders included BPPV and vestibulo-ocular reflex disorder each in three (3.33\%) patients, and Meniere's disease, meningitis, and phobic postural vertigo in $2(2.22 \%)$ patients each. The remaining disorders included congenital nystagmus, drug-induced dizziness caused by antiepileptics, vestibulospinal dysfunction, otolithic drop attacks, epilepsy, psychogenic vertigo, labyrinthitis, and autoimmune vestibulopathy in one (1.11\%) patient. - Table 2 shows the symptoms this study participants presented on their first visit. The duration of these symptoms varies from days to years. - Fig. $\mathbf{2}$ shows in detail the duration of the symptoms observed in the participants.

Table 1 Characteristics of this study participants

\begin{tabular}{|l|l|}
\hline Patient's characteristics on presentation & \\
\hline Total number of patients & 90 \\
\hline Male/Female & $56 / 34$ \\
\hline $\begin{array}{l}\text { Age }(y) \text { at presentation (mean } \pm \text { standard } \\
\text { deviation [SD]) }\end{array}$ & $10.67 \pm 4.35$ \\
\hline Age range $(y)$ & $3-16$ \\
\hline
\end{tabular}

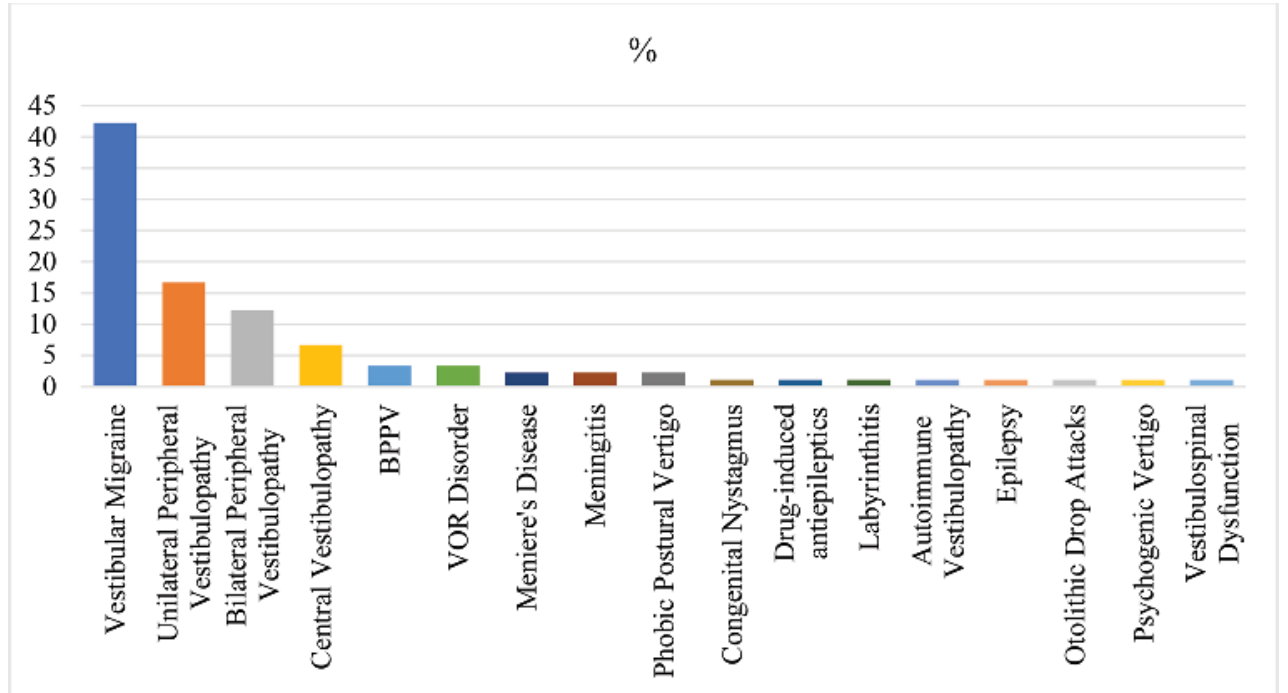

Fig. 1 Distribution of the disorders causing vertigo in the study participants. BPPV, benign paroxysmal positional vertigo; VOR, vestibuloocular reflex. 
Table 2 Symptoms of the study participants when they first visited the clinic

\begin{tabular}{|l|l|l|}
\hline $\begin{array}{l}\text { Symptoms at } \\
\text { presentation }\end{array}$ & No. of patients & Percentage \\
\hline Headache & 50 & 55.6 \\
\hline Nausea & 38 & 42.2 \\
\hline Loss of consciousness & 9 & 10 \\
\hline Hearing loss & 28 & 31.1 \\
\hline Tinnitus & 12 & 13.3 \\
\hline
\end{tabular}

-Fig. 3 shows the age distribution according to cause of vertigo in this study participants. Vestibular migraine, previously termed benign paroxysmal vertigo of childhood (BPVC), was seen to be the most common cause of vertigo in children between 7 to 16 years of age. Unilateral and bilateral peripheral vestibulopathy cases were seen more commonly in children between 3 and 6 years of age, whereas central vestibulopathy was seen in children of all ages.
During the vestibular examination, various abnormalities were seen. A vertical down-beating nystagmus was seen in a patient on antiepileptic drug lamotrigine ( - Fig. 4). After revising the dose of the drug, unsteadiness and nystagmus were resolved. Patients with BPPV of the posterior canal showed nystagmus during Dix-Hallpike test (-Fig. 5). Patients with Meniere's disease showed abnormal sway during craniocorpography (-Fig. 6).

\section{Discussion}

Harrison in 1962 reported the first case of peripheral cause of vertigo in children. ${ }^{2}$ This was followed by Eviatar and Eviatar in 1977 who reported a predominance of central pathology such as vertiginous seizures in children and adolescents. $^{8}$ Fried $^{9}$ and D'Agostino et al $^{10}$ discussed cerebral concussion causing childhood vertigo. Bower and Cotton in 1995 reported peripheral causes of vertigo. ${ }^{11}$ Otitis media shows apparent symptoms of infection, making it very easy

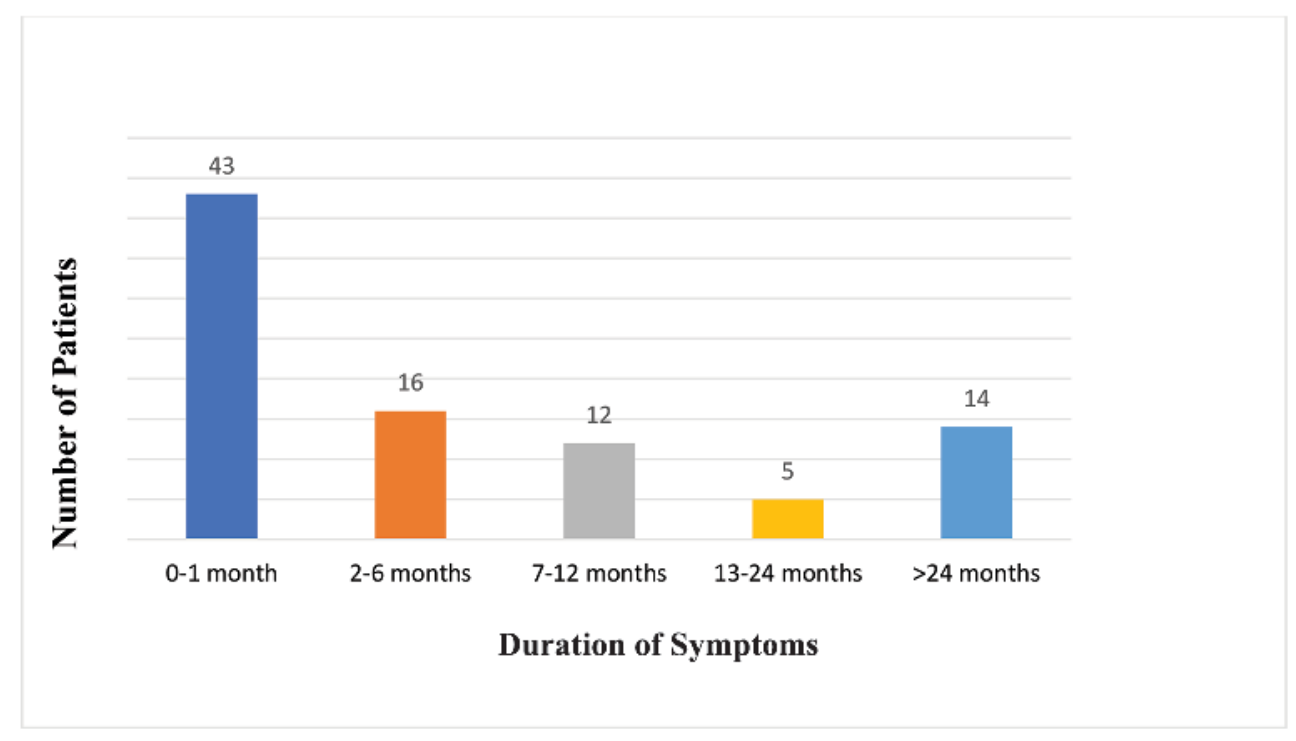

Fig. 2 Duration of the symptoms.

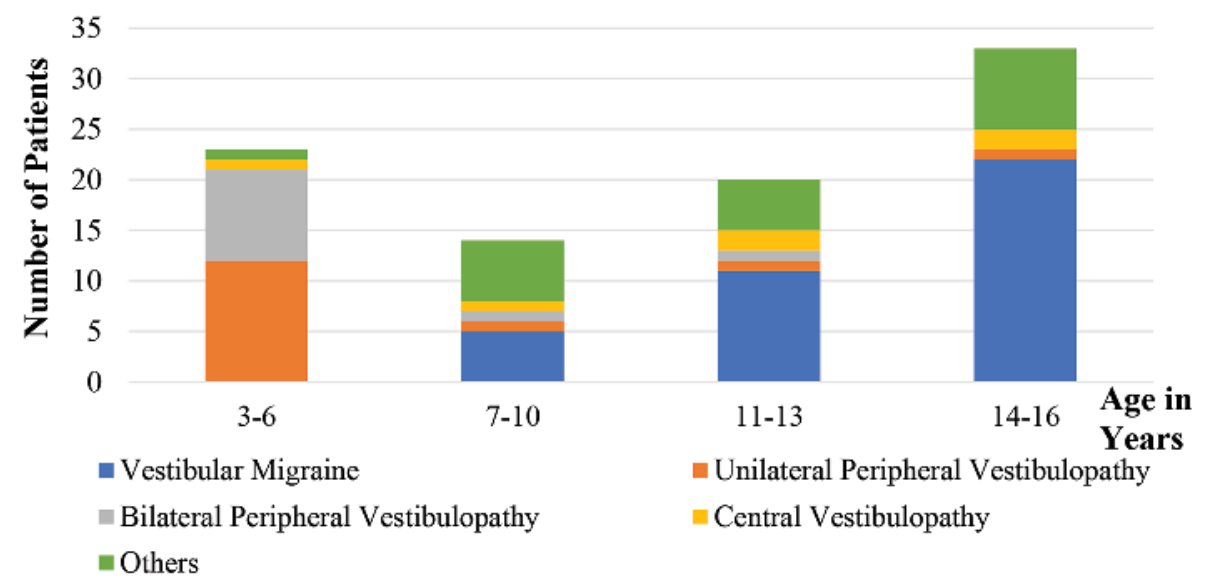

Fig. 3 Age-wise distribution of the cause of vertigo in the study participants. 
24 Differential Diagnosis of Vertigo in Children Bhandari, Goswami

\begin{tabular}{|lllll}
\hline Nystagmus Direction & Right Eye SPV (/sec) & Right Eye Beats $/ 30$ sec & Left Eye SPV $(/ \mathrm{sec})$ & Left Eye Beats $/ 30 \mathrm{sec}$ \\
\hline Horizontal Left Beating & 0 & 0 & 0 & 0 \\
\hline Horizontal Right Beating & 0 & 0 & 0 & 0 \\
\hline Vertical Up Beating & 0 & 0 & 0 & 0 \\
\hline Vertical Down Beating & 4 & 11 & 4 & 16 \\
\hline
\end{tabular}
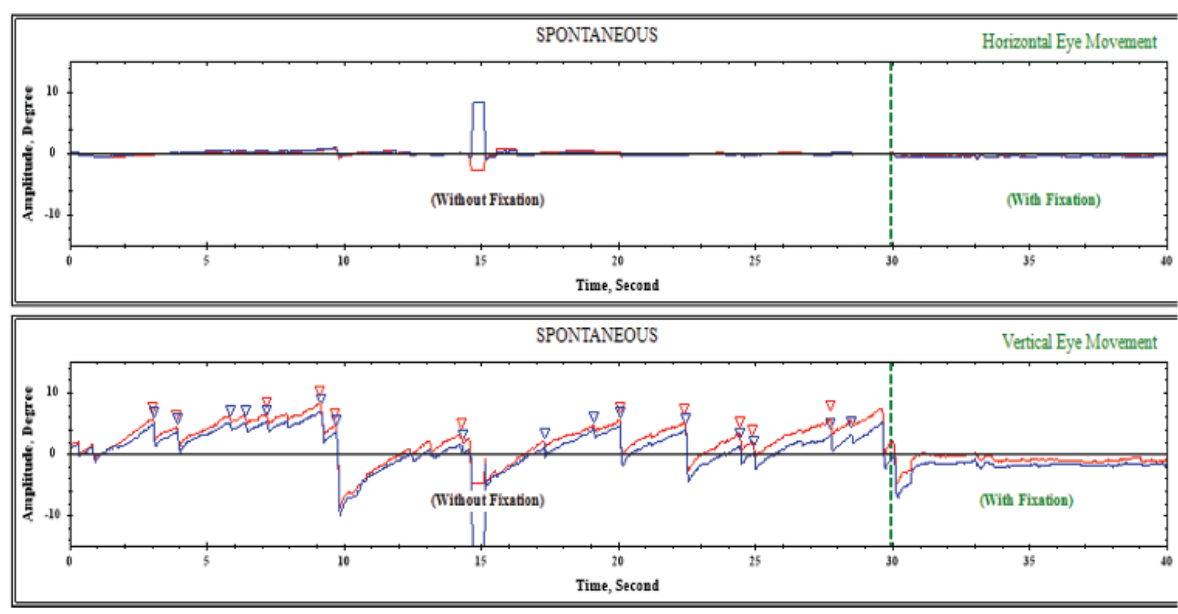

Fig. 4 Spontaneous vertical down-beating nystagmus due to lamotrigine. SPV, slow-phase velocity.

\begin{tabular}{|lllll|}
\hline & Right Eye SPV( $/ \mathrm{sec})$ & Right Eye Beats & Left Eye SPV( $/ \mathrm{sec})$ & Left Eye Beats \\
\hline Dix-Hallpike Right Horizontal & 0 & 0 & 0 & 0 \\
\hline Dix-Hallpike Right Vertical & 0 & 0 & 0 & 0 \\
\hline Dix-Hallpike Left Horizontal & 0 & 0 & 0 & 0 \\
\hline Dix-Hallpike Left Vertical & -5 & 36 & -10 & 75 \\
\hline
\end{tabular}
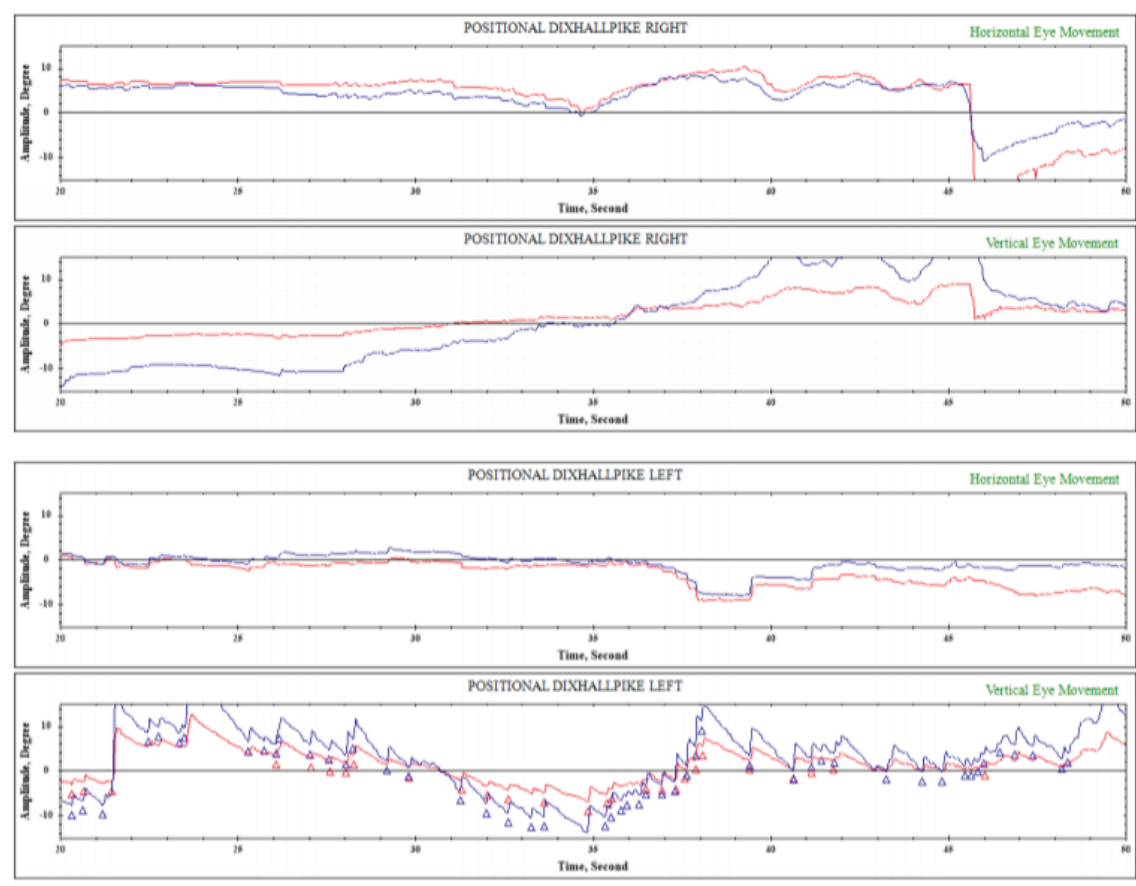

Fig. 5 Nystagmus in left Dix-Hallpike test in a patient with benign paroxysmal positional vertigo, BPPV; benign paroxysmal positional vertigo; SPV, slow-phase velocity. 

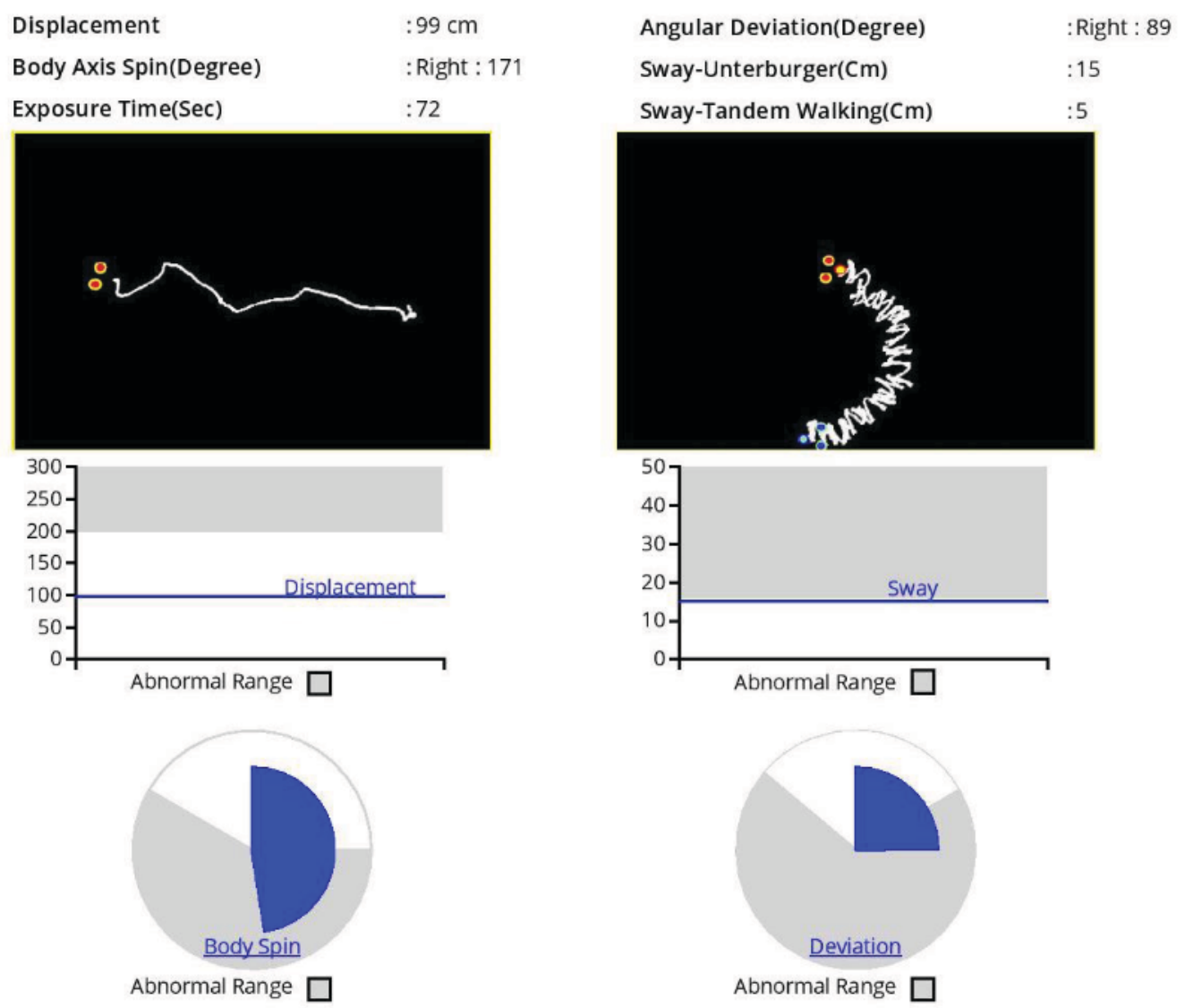

Fig. 6 Abnormal sway seen on craniocorpography (CCG) in a patient with Meniere's disease.

to diagnose. Because we had excluded the cases of otitis media, vestibular migraine was found to be the most common of cause of vertigo in children. There are many other studies that showed migraine-associated vertigo in most participants of this study (children and adolescents). ${ }^{12-15} \mathrm{In}$ a study by Golz et al, middle ear effusion was seen in around $58 \%$ of the study population. ${ }^{16}$ In our study, the most common disorder was found to be vestibular migraine (42.2\%). In adults, vestibular migraine and BPPV are seen to be the most frequent causes of vertigo. Meniere's disease shows a median time of onset of 40 years. It is quite uncommon in children, but we found two patients with this disorder in our study. Meniere's disease may present with vomiting in children, which may be misdiagnosed as cyclic vomiting of migraine. ${ }^{17}$ BPPV is also a rare finding in children. In this study, two patients were seen to have position-triggered vertigo associated with BPPV. Some of the causes of vertigo may remain unclassified. Studies report that those cases could be basilar migraine. ${ }^{14} \mathrm{~A}$ study focusing on unexplained neurological complaints seen in children with vertigo, headache, dizziness, or fainting showed that at least one psychiatric disorder was seen in more than $90 \%$ of the participants. ${ }^{18}$

Our study showed various etiologies leading to symptoms of vertigo in children. Detailed anamnesis along with comprehensive otoneurological examination helps achieve correct diagnosis and treatment plan. The differences in the result seen in our study in comparison to other published literature can be attributed to the study design and the criteria for inclusion and exclusion. Our study included patients complaining of vertigo and dizziness only. Children with acute or chronic otitis media and with previously known neurological deficits were excluded from the study.

Videonystagmography (VNG) has been used increasingly as the workhorse in the otoneurologist armamentarium. All the study participants completed the VNG examination. Valente, in her study, stated that for determining the underlying causes of vertigo and children, bedside clinical testing and accurate patient history along with laboratory diagnosis and vestibular examination are required to arrive at and ascertain final diagnosis. ${ }^{19}$

\section{Conclusion}

Vertigo in children must be recognized as a definite symptom, and its evaluation is necessary to ascertain the underlying etiology and provide the correct treatment. Although vestibular migraine is the most common cause of vertigo in children, other etiologies for pediatric vertigo are myriad. Meticulous history and evaluation are the only way to get an insight into the various etiologies for childhood vertigo and thereby achieve a successful treatment for the same. Proactive interdisciplinary follow-up of patients avoids incorrect or undertreatment and misdiagnosis that may lead to the deterioration of the quality of life. 


\section{Funding}

None.

\section{Conflict of Interest}

None declared.

\section{References}

1 MacGregor DL. Vertigo. Pediatr Rev 2002;23(1):10-16

2 Harrison MS. Vertigo in childhood. J Laryngol Otol 1962;76(8):601-616

3 Gruber M, Cohen-Kerem R, Kaminer M, Shupak A. Vertigo in children and adolescents: characteristics and outcome. Scientific World Journal 2012;2012:109624

4 Walls TJ, Teach S, Evaluation of dizziness in children and adolescents UpToDate. Uptodate.com. 2017. Available at: https:// www.uptodate.com/contents/evaluation-of-dizziness-in-children-and-adolescentsAccessed February 7, 2019

5 Casselbrant ML, Furman JM, Rubenstein E, Mandel EM. Effect of otitis media on the vestibular system in children. Ann Otol Rhinol Laryngol 1995;104(8):620-624

6 Benun J. Balance and vertigo in children. Pediatr Rev 2011;32(2):84-85

7 Balatsouras DG, Kaberos A, Assimakopoulos D, Katotomichelakis M, Economou NC, Korres SG. Etiology of vertigo in children. Int J Pediatr Otorhinolaryngol 2007;71(3):487-494

8 Eviatar L, Eviatar A. Vertigo in children: differential diagnosis and treatment. Pediatrics 1977;59(6):833-838

9 Fried MP. The evaluation of dizziness in children. Laryngoscope 1980;90(9):1548-1560
10 D’Agostino R, Tarantino V, Melagrana A, Taborelli G. Otoneurologic evaluation of child vertigo. Int J Pediatr Otorhinolaryngol 1997;40(2-3):133-139

11 Bower CM, Cotton RT. The spectrum of vertigo in children. Arch Otolaryngol Head Neck Surg 1995;121(8):911-915

12 Choung YH, Park K, Moon SK, Kim CH, Ryu SJ. Various causes and clinical characteristics in vertigo in children with normal eardrums. Int J Pediatr Otorhinolaryngol 2003;67(8):889-894

13 O'Reilly RC, Greywoode J, Morlet T, et al. Comprehensive vestibular and balance testing in the dizzy pediatric population. Otolaryngol Head Neck Surg 2011;144(2):142-148

14 Wiener-Vacher SR. Vestibular disorders in children. Int J Audiol 2008;47(9):578-583

15 Erbek SH, Erbek SS, Yilmaz I, et al. Vertigo in childhood: a clinical experience. Int J Pediatr Otorhinolaryngol 2006;70(9):1547-1554

16 Golz A, Netzer A, Angel-Yeger B. Westerman ST, Gilbert LM, Joachims HZ. Effects of middle ear effusion on the vestibular system in children. Otolaryngol Head Neck Surg 1998;119(6):695-699

17 Miyahara M, Hirayama M, Yuta A, Takeuchi K, Inoki T. Too young to talk of vertigo? Lancet 2009;373(9662):516

18 Emiroğlu FN, Kurul S, Akay A, Miral S, Dirik E. Assessment of child neurology outpatients with headache, dizziness, and fainting. J Child Neurol 2004;19(5):332-336

19 Valente LM. Assessment techniques for vestibular evaluation in pediatric patients. Otolaryngol Clin North Am 2011;44(2):273-290 